\title{
MEASURING THE HUBBLE CONSTANT WITH THE HUBBLE SPACE TELESCOPE $\dagger$
}

\author{
W. L. Freedman, R. C. Kennicutt \& J. R. Mould
}

\begin{abstract}
Ten years ago our team completed the Hubble Space Telescope Key Project on the extragalactic distance scale. Cepheids were detected in some 25 galaxies and used to calibrate four secondary distance indicators that reach out into the expansion field beyond the noise of galaxy peculiar velocities. The result was $\mathrm{H}_{0}=72 \pm 8 \mathrm{~km} \mathrm{~s}^{-1} \mathrm{Mpc}^{-1}$ and put an end to galaxy distances uncertain by a factor of two. This work has been awarded the Gruber Prize in Cosmology for 2009.
\end{abstract}

\section{Introduction}

Our story begins in the mid-1980s with community-wide discussions on Key Projects for the NASA/ESA Hubble Space Telescope. The extragalactic distance scale was the perfect example of a project that would be awarded a generous allocation of time in order that vital scientific goals would be accomplished even if the lifetime of the telescope proved to be short. As Marc Aaronson (Figure 1), the original principal investigator of the project, said in his Pierce Prize Lecture in 1985, "The distance scale path has been a long and torturous one, but with the imminent launch of HST there is good reason to believe that the end is finally in sight."

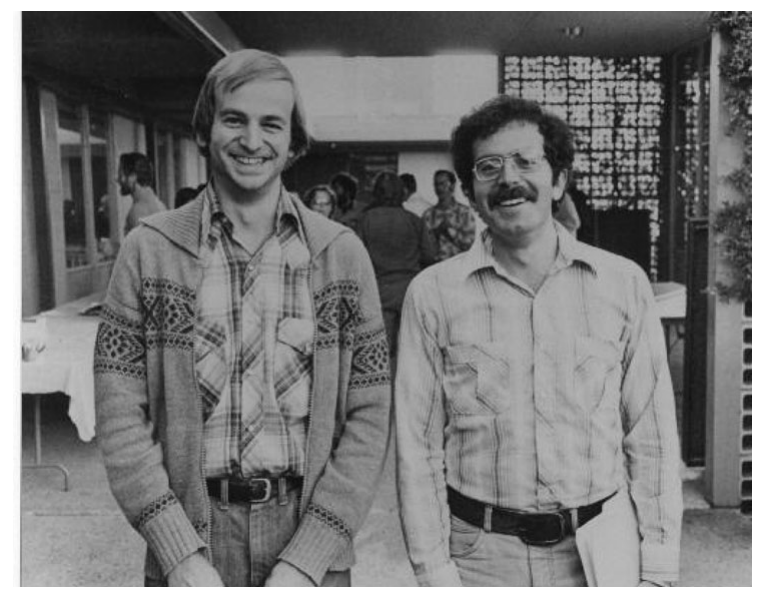

Figure 1. Jeremy Mould (left) and Marc Aaronson (right) at the van Biesbroeck Prize award ceremony in 1981.

Marc Aaronson died tragically in an accident in 1987, having written a successful proposal for the Key Project, a project designed to shrink the scatter shown in Figure 2 to $10 \%$ and put an end to 60 years of debate, commencing with Hubble's estimates in 1929.

$\dagger$ Based on observations made with the NASA/ESA Hubble Space Telescope obtained at the Space Telescope Science Institute which is operated by AURA for NASA. 


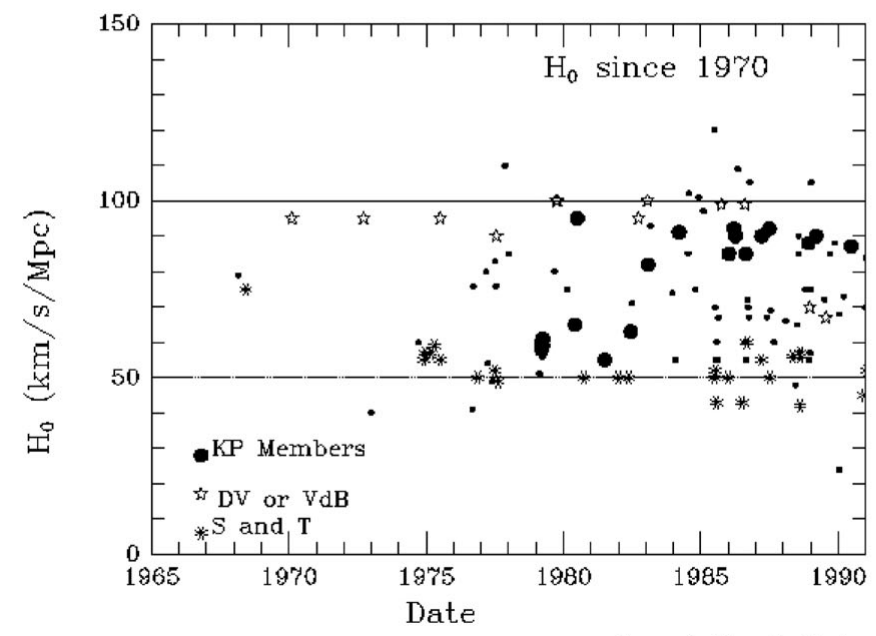

Copuright J. Huchra 2008

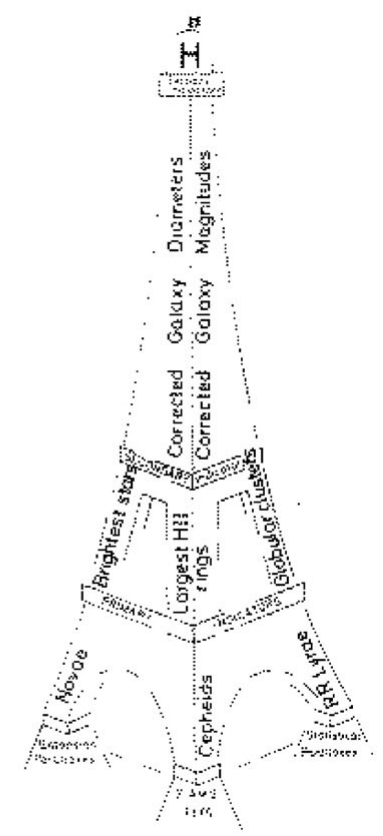

Figure 2. The scatter in estimates of the Hubble Constant showed no sign of convergence in the 1970s and 1980s. On the right is a schematic distance scale by G. de Vaucouleurs, one of the protagonists in the distance scale controversy.

The principal reason for the uncertainty in $\mathrm{H}_{0}$ is evident in Figure 3. Large scale structure is seen out to distances of $100 \mathrm{Mpc}$. Ground-based Cepheid distances, however, extended to only $4 \mathrm{Mpc}$ with a "twilight zone" beyond (Figure 4).

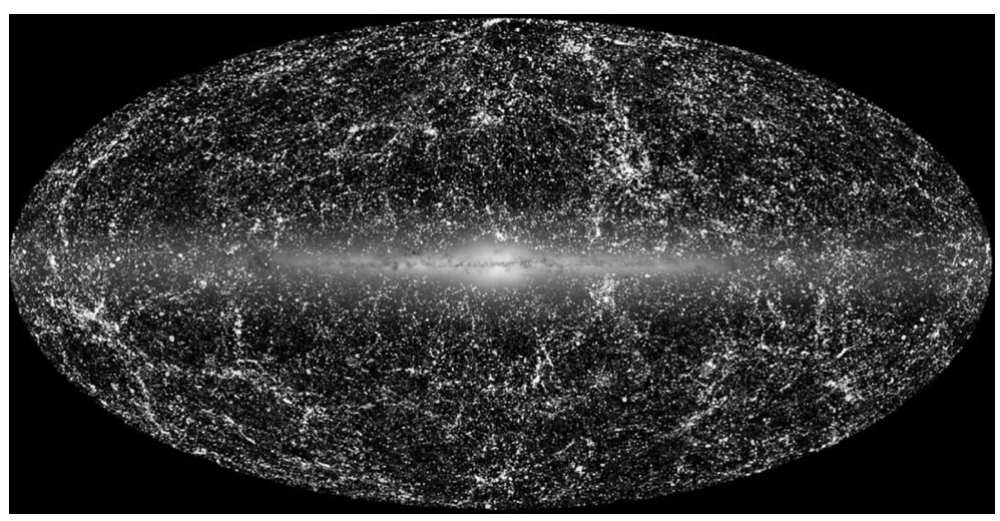

Figure 3. Colour coded redshifts show the large scale structure in the nearby Universe (galactic coordinates).

The Key Project's solution to the twilight zone problem was to map Cepheids out to $20 \mathrm{Mpc}$ and calibrate secondary distance indicators within this volume. The secondary indicators would extend the distance scale out to $100 \mathrm{Mpc}$. 
ALLAN SANDAGE AND G. A. TAMMANN
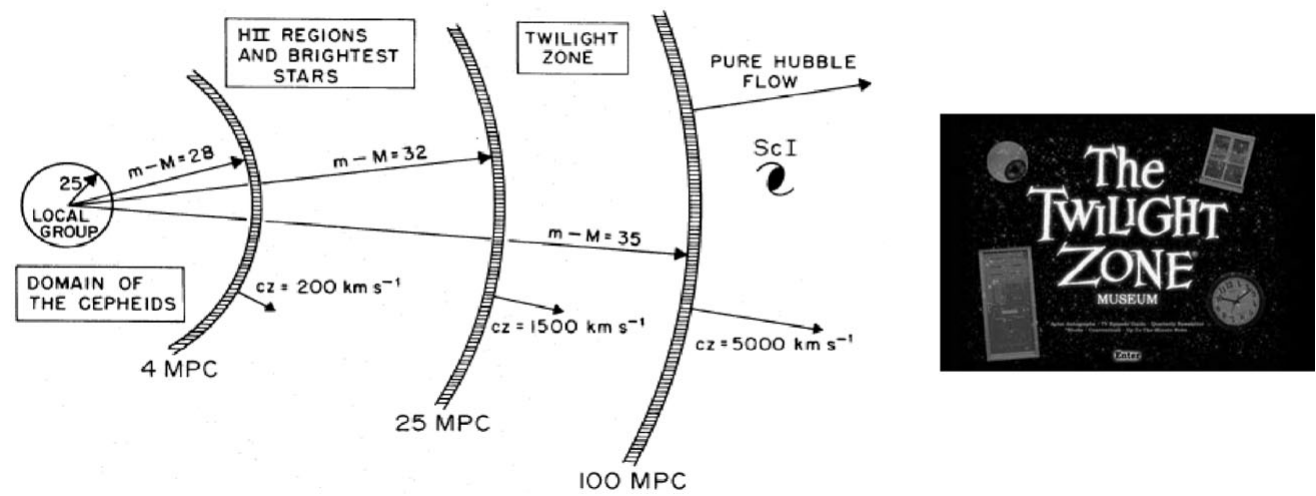

Figure 4. A schematic distance scale by other protagonists of the controversy, A. Sandage \& G. Tammann.

An important tenet of the Key Project was to exploit the redundancy of distance indicators, as shown in Figure 5, especially the four secondary distance indicators, the Tully-Fisher relation, surface brightness fluctuations, supernovae, and the fundamental plane.

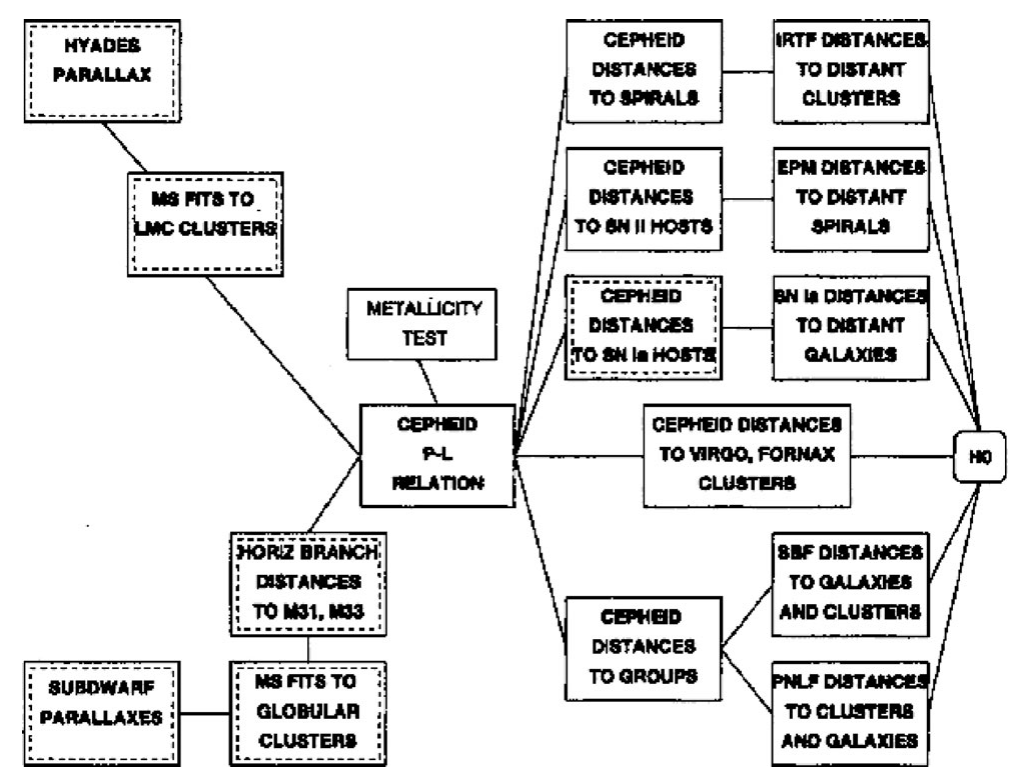

Figure 5. The schematic distance scale in the Key Project Committee report.

\section{Cepheids}

The Cepheid Period-Luminosity law was discovered by Leavitt early in the twentieth century.

By the end of the century much was understood about the systematics of Cepheids. For example (Madore \& Freedman 1991), Cepheid amplitudes are maximum in the blue, and interstellar absorption is minimum at long wavelengths (Figure 7). Therefore, the best strategy for discovering Cepheids is to observe at visible wavelengths; to minimize the effect of dust, luminosities are best measured in the infrared. 

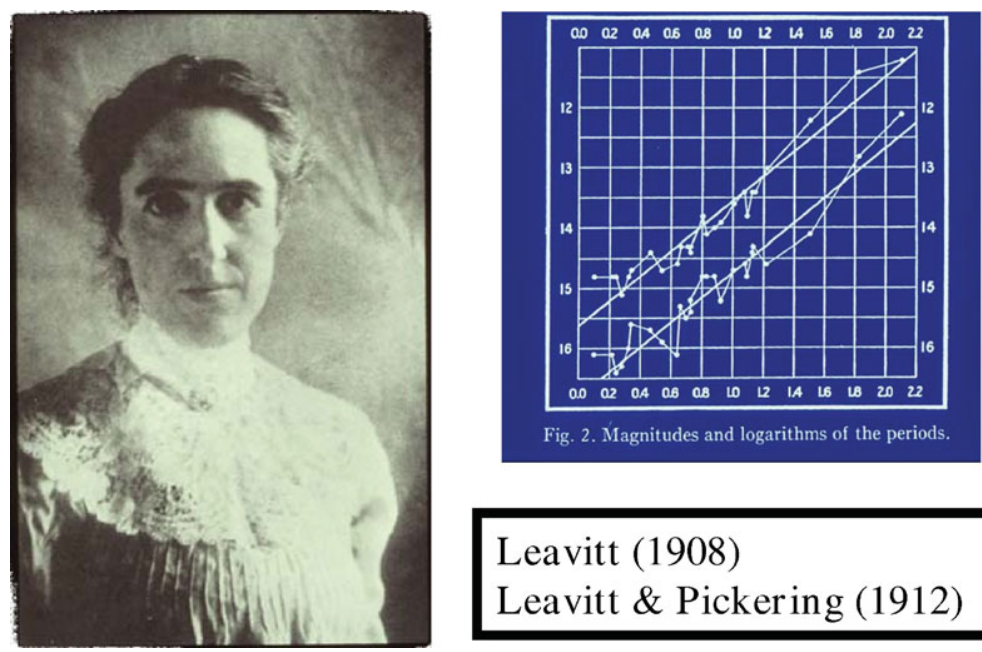

Leavitt (1908)

Leavitt \& Pickering (1912)

Figure 6. Henrietta Leavitt and the period-luminosity relation in the Magellanic Clouds.

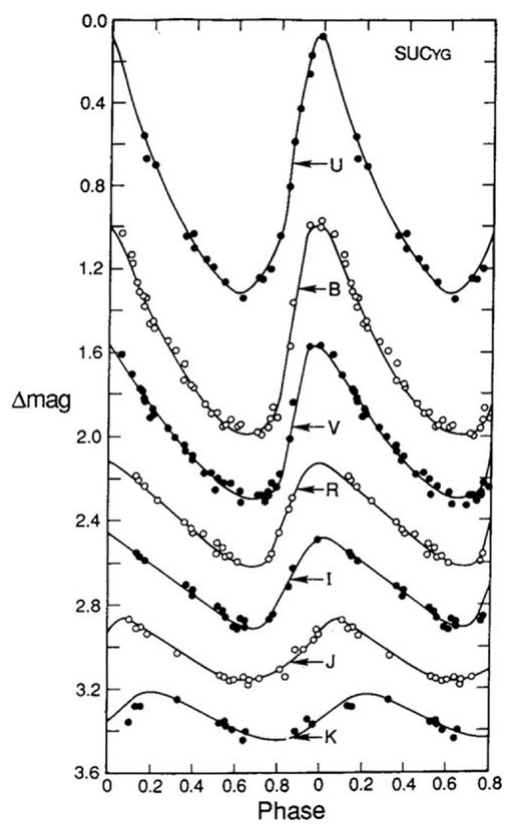

Figure 7. Amplitude as a function of wavelength for Cepheid variables.

HST brought a number of strengths to the Cepheid distance scale: linear detectors, multiwavelength observations, and a planned cadence of observations. Figure 8 shows for M33 (Freedman et al. 1991) how an absolute distance modulus is obtained with knowledge of the reddening law.

Figure 9 shows how a power law observing cadence is superior for luminosity measurement to equally spaced observations (Madore \& Freedman 2002, 2005).

The results are shown in Figure 10. The light curves for these periods are unmistakably Cepheids. Our observations populated the range 10-100 days in galaxies with distances of order $10 \mathrm{Mpc}$.

Figure 11 shows a typical field placement for the Key Project in the large face-on spiral galaxy in the Virgo cluster, M100. Twelve V observations were obtained during the sequence and four I observations. Cosmic ray splits were used and a fixed roll angle was adopted. Photometry was 


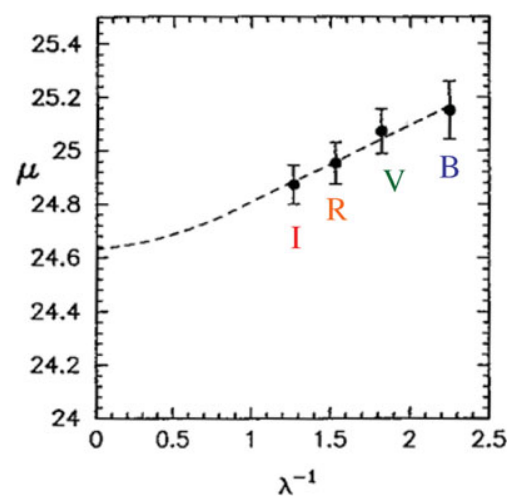

Figure 8. Finding the distance of M33 (Freedman et al. 1991).

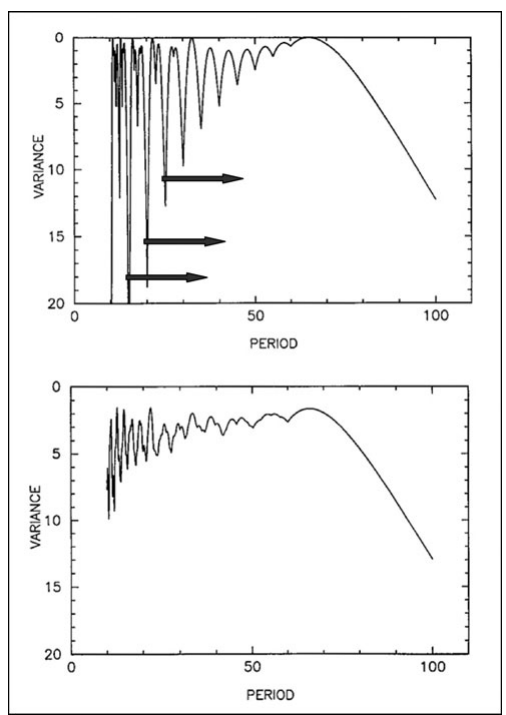

Figure 9. Observing cadence. Upper panel: equally spaced observations. Lower panel: power law cadence.

carried out on the frames using DoPhot and a custom version of DAOPHOT called ALLFRAME (Stetson 1994).

A composite I-band period-luminosity relation for 800 Cepheids in 24 galaxies is shown in Figure 12 (corrected for distance) (Ferrarese et al. 2000).

Ferrarese et al. (2000) also carried out a comprehensive comparison of Key Project distances with other prominent distance indicators, such as the tip of the red giant branch (e.g. Sakai et al. 1997) and surface brightness fluctuations (Tonry et al. 2001) (Figure 13) and the planetary nebula luminosity function (Ciardullo \& Jacoby 1992) and the globular cluster luminosity function (Secker \& Harris 1993) (Figure 14).

\section{Metallicity Calibration}

The Cepheid period luminosity relation is a straightforward primary distance indicator if the precepts described above are followed. But there is a complication. Cepheids vary in their chemical composition, and the period luminosity relation is affected. We can write

$$
(m-M)_{\text {true }}=(m-M)_{P L}-\gamma \log \left(Z / Z_{L M C}\right)
$$

where $Z$ is the metallicity of the field and $Z_{L M C}$ is the metallicity of the LMC.

However, theory is not predictive, even about the sign of $\gamma$. According to Chiosi, Wood \& 


\section{NGC 4414}
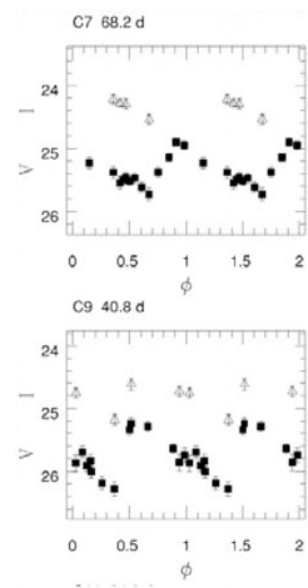
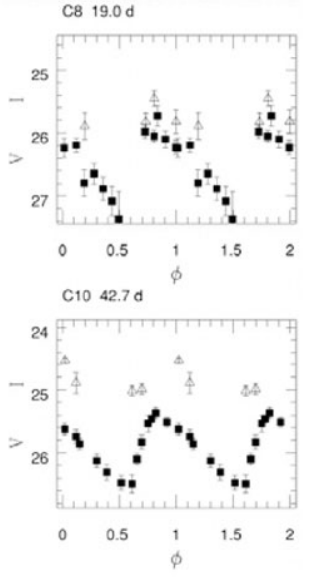

NGC 2090
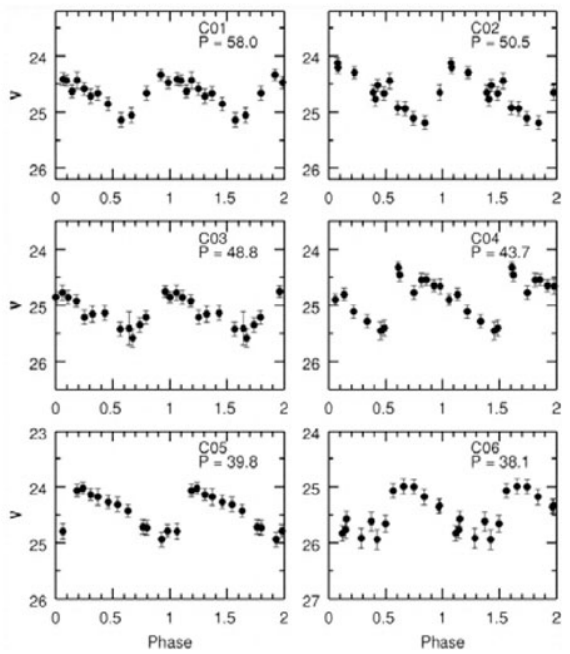

Figure 10. Light curves for Cepheids in NGC 2090 (Phelps et al. 1998) and NGC 4414 (Turner et al. 1998).

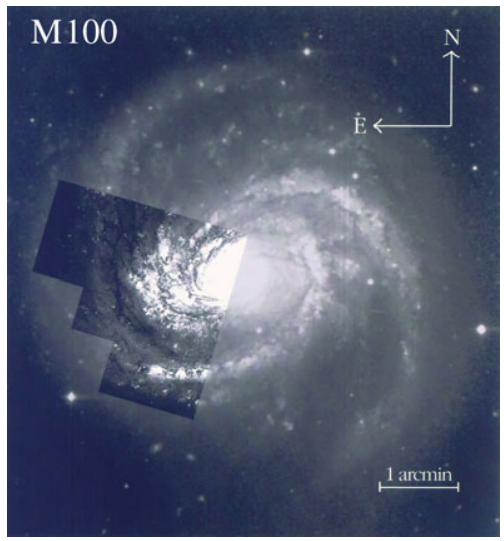

Figure 11. The WFPC2 footprint is shown on an image of M100.

Capitanio (1993) $\gamma_{V I}=-0.11 \mathrm{mag} / \mathrm{dex}$, but elsewhere we find with different opacities $\gamma_{V I}=$ $+0.06 \mathrm{mag} / \mathrm{dex}$. The Key Project took an empirical approach, described by Kennicutt (1998). Both an inner and an outer field were observed in M101 (Figure 15), a galaxy with a large abundance gradient (Figure 16). The Cepheid metallicities were assumed to follow the oxygen abundances of nearby HII regions. The inner field modulus was found to be $29.20 \pm 0.09 \mathrm{mag}$, and the outer modulus was $29.36 \pm 0.08 \mathrm{mag}$. There was a factor of five difference in $\mathrm{Z}$. This yielded $\gamma=-0.24 \pm 0.16$. Oxygen abundances were measured for each of the Key Project galaxies by Zaritsky, Kennicutt, and Huchra (1994).

Confirmation of this result comes from a comparison of tip of the red giant branch distances (TRGB) and Cepheid distances by Ferrarese et al. (2000) (Figure 17). 


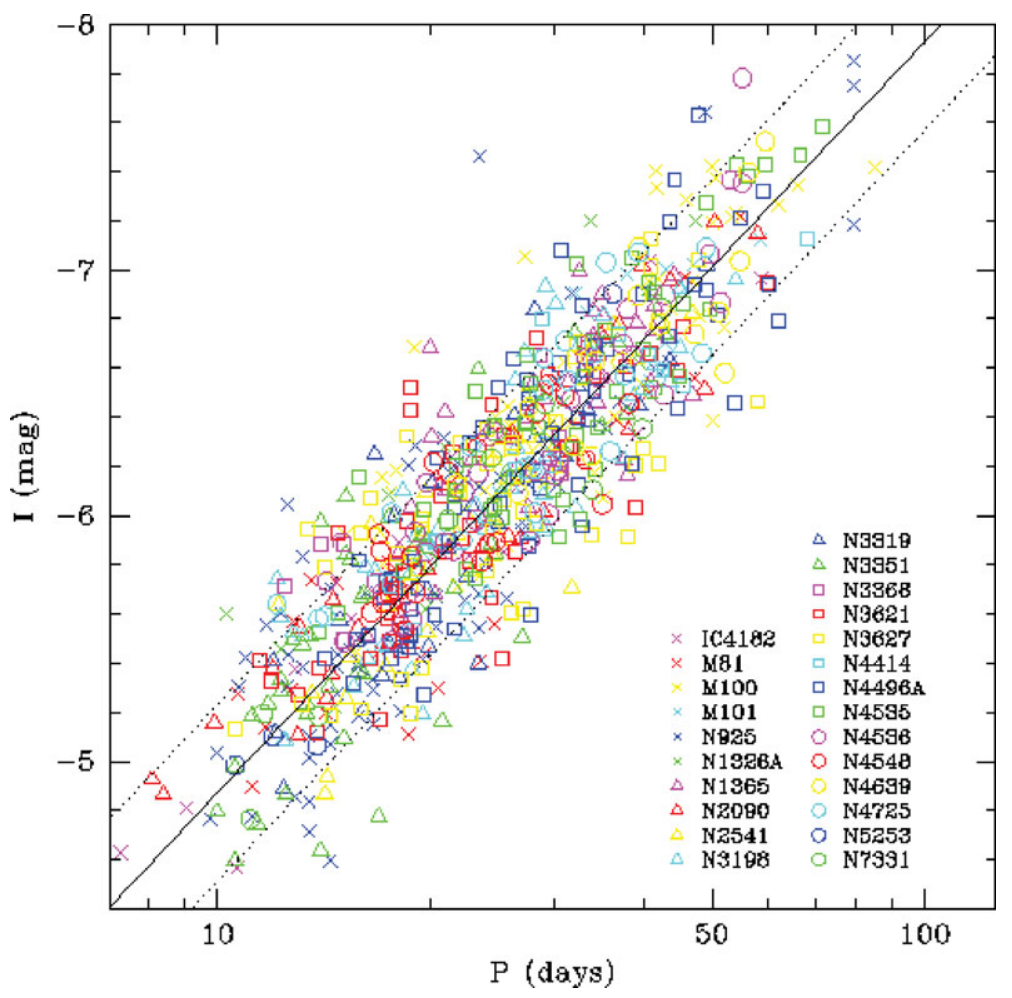

Figure 12. Composite I-band PL relation for the Key Project.
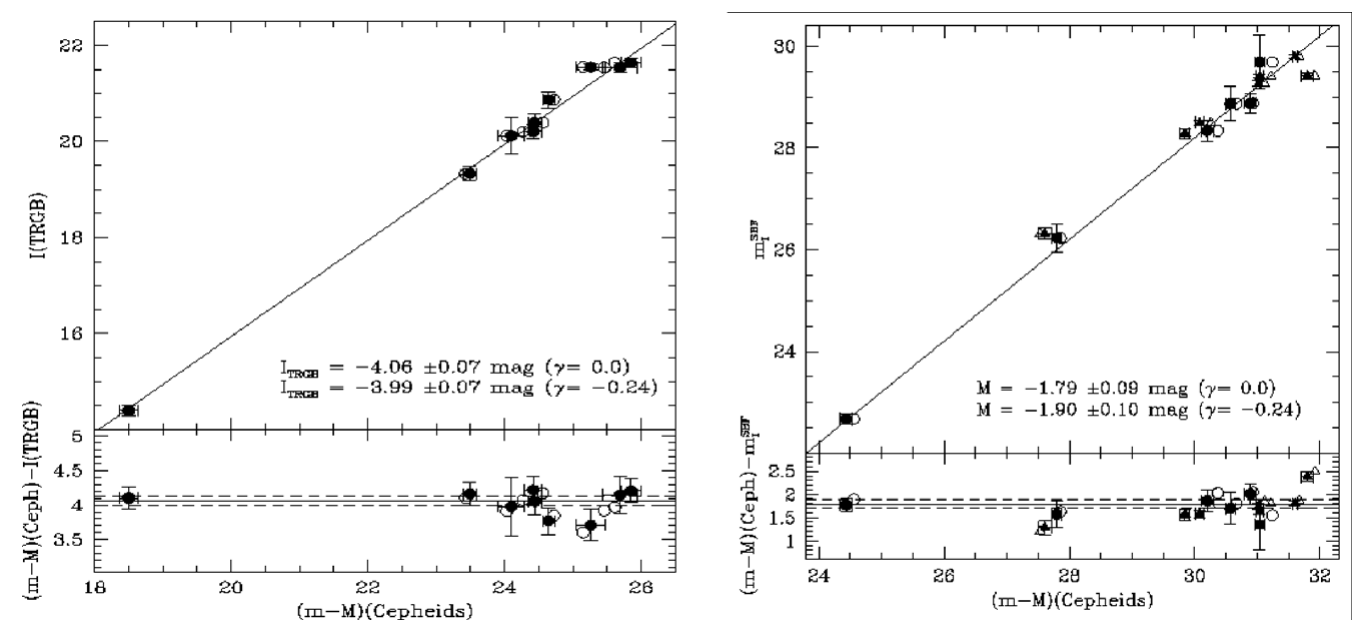

Figure 13. Comparison of Cepheid distances with the tip of the red giant (left) and surface brightness fluctuations (right).

More recent work has strengthened these results. Mould \& Sakai (2008, 2009ab) have shown that substitution of TRGB distances for Cepheid distances in secondary distance indicator calibrations returns a Hubble Constant in agreement with the Key Project. And Scowcroft et al. (2009) obtained $\gamma_{V I}=-0.26$ in a study of M33. 

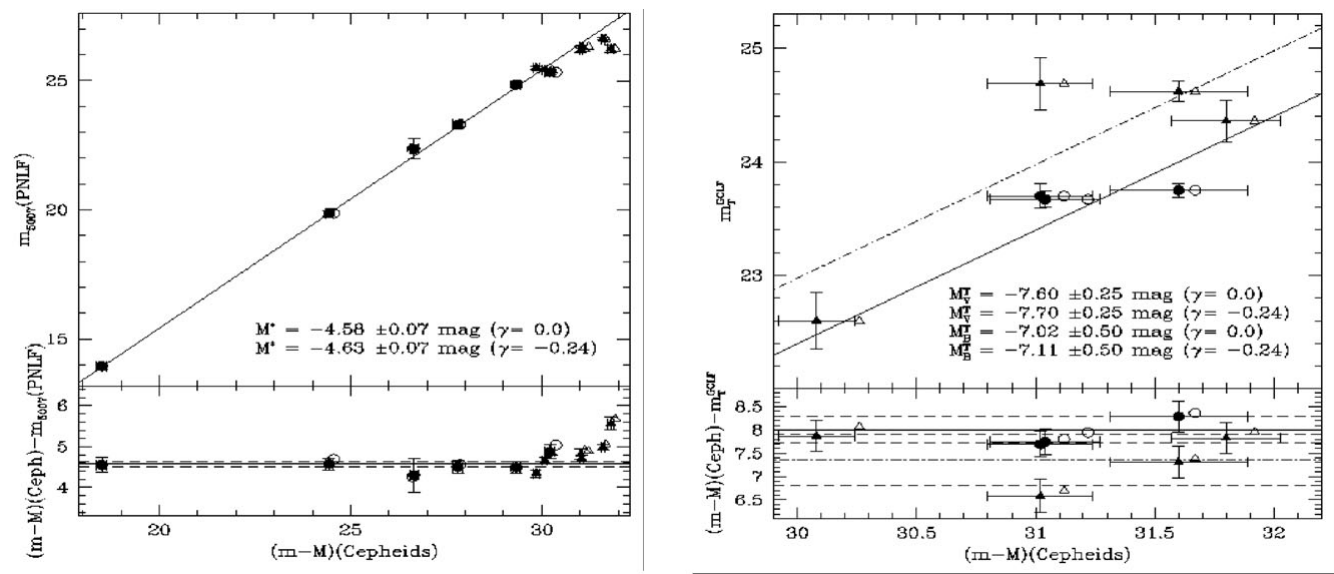

Figure 14. Comparison of Cepheid distances with the planetary nebula luminosity function (left) and the globular cluster luminosity function (right).

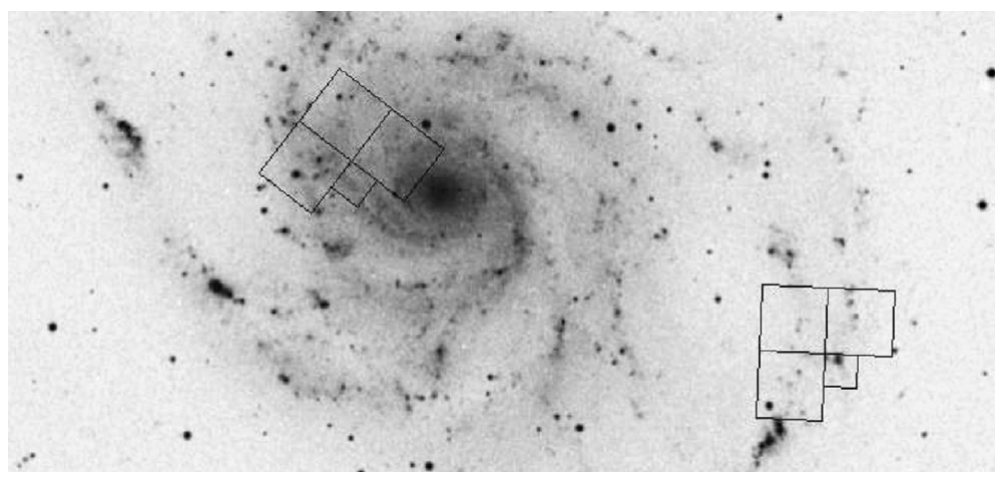

Figure 15. The two WFPC2 fields observed in M101.

\section{Measurement of the Hubble Constant}

Four secondary distance indicators were calibrated by the Key Project. The first was the TullyFisher relation (Figure 18). Sakai et al. (2000) obtained $\mathrm{H}_{0}=71 \pm 3 \pm 7 \mathrm{~km} \mathrm{~s}^{-1} \mathrm{Mpc}^{-1}$, where the first uncertainty is the random error and the second uncertainty is the systematic error.

Next comes the fundamental plane. Kelson et al. (2000) used Cepheid distances to the Leo, Virgo, and Fornax clusters to calibrate the fundamental plane and the $\mathrm{D}_{n}, \sigma$ relation (Figure 20), obtaining $\mathrm{H}_{0}=78 \pm 5 \pm 9 \mathrm{~km} \mathrm{~s}^{-1} \mathrm{Mpc}^{-1}$.

Type Ia supernovae were calibrated by Gibson et al. (2000), using 6 supernova hosts with measured decline rates, some of them reworked from Saha et al. (1999). Figure 21 shows the application of the calibration to supernovae out to $30,000 \mathrm{~km} \mathrm{~s}^{-1}$, yielding $\mathrm{H}_{0}=71 \pm 2 \pm$ $7 \mathrm{~km} \mathrm{~s}^{-1} \mathrm{Mpc}^{-1}$.

Finally, Ferrarese et al. (2000a) calibrated surface brightness fluctuations in early type galaxies, obtaining $\mathrm{H}_{0}=69 \pm 4 \pm 6 \mathrm{~km} \mathrm{~s}^{-1} \mathrm{Mpc}^{-1}$.

A good summary of the results is provided by Figure 22, which shows the Cepheids and the calibrated distance indicators to a redshift of 0.1 , well beyond the effect of local velocity field perturbations. Hubble's (1929) distances are confined to the first tick mark in Figure 23.

The uncertainties in the Hubble Constant remain dominated by systematic errors. The first of these is reddening, and this has been tested in work by Macri et al. (2001), who reobserved many of the Key Project Cepheids with the NICMOS infrared camera. Their results for M81 are shown in Figure 24. Overall, the $\mathrm{H}$ band distances agreed with the Key Project distances to $1 \%$. 


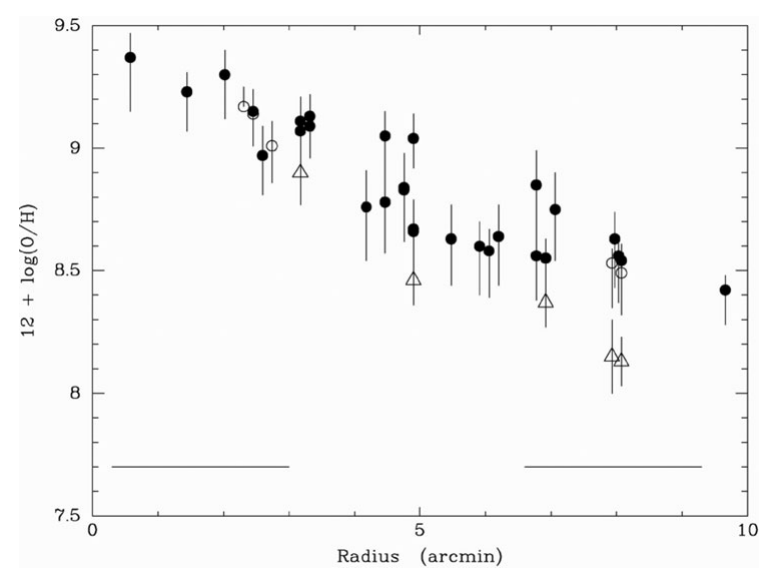

Figure 16. The gradient in oxygen abundance in M101.

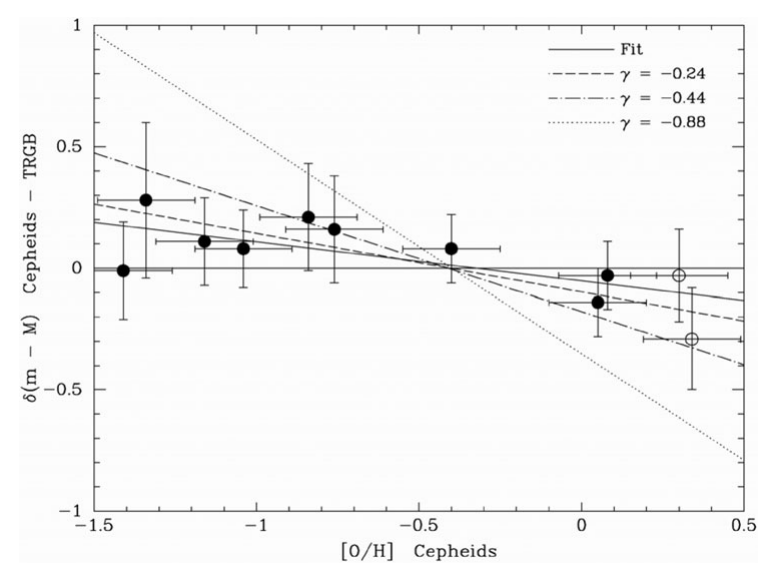

Figure 17. Comparison of TRGB and Cepheid distances for different values of $\gamma$.

We have dealt with the second systematic error, metallicity differences with the Large Magellanic Cloud (LMC), but the LMC remains the principal systematic error because of the assumption of its distance modulus, 18.50. A second fiducial distance has subsequently become available in the maser distance of NGC 4258. Herrnstein et al. (1999) obtained the distance of this galaxy by fitting a simple kinematic model to the maser radial velocities and VLBI proper motions $(7.3 \pm 0.4 \mathrm{Mpc}$ ). The Cepheid distance is $7.5 \pm 0.3 \mathrm{Mpc}$ (Macri et al. 2006). In addition, HST trigonometric parallaxes for a sample of Cepheids have become available (Benedict et al. 2007), confirming the Key Project period-luminosity calibration, as shown in Figure 25.

The probability distribution for $\mathrm{H}_{0}$, combining the results of the secondary distance indicators and the full error budget of the Key Project is shown in Figure 26 (Freedman et al. 2001).

\section{The Team}

Most of the team members (many referenced above) are captured in Figure 27. Special roles were played by the individuals depicted in Figure 28 and 29.

Other notable contributions were made by John Graham, Nancy Silbermann, Randy Phelps, Daya Rawson, Fabio Bresolin, Lucas Macri, Bob Hill, Kim Sebo, Paul Harding, Anne Turner, Han Ming Sheng, Shaun Hughes, Charles Prosser, John Huchra, Holland Ford, and Garth Illingworth. Jim Gunn, Sandra Faber and John Hoessel were instrument team liaisons. The team drew 

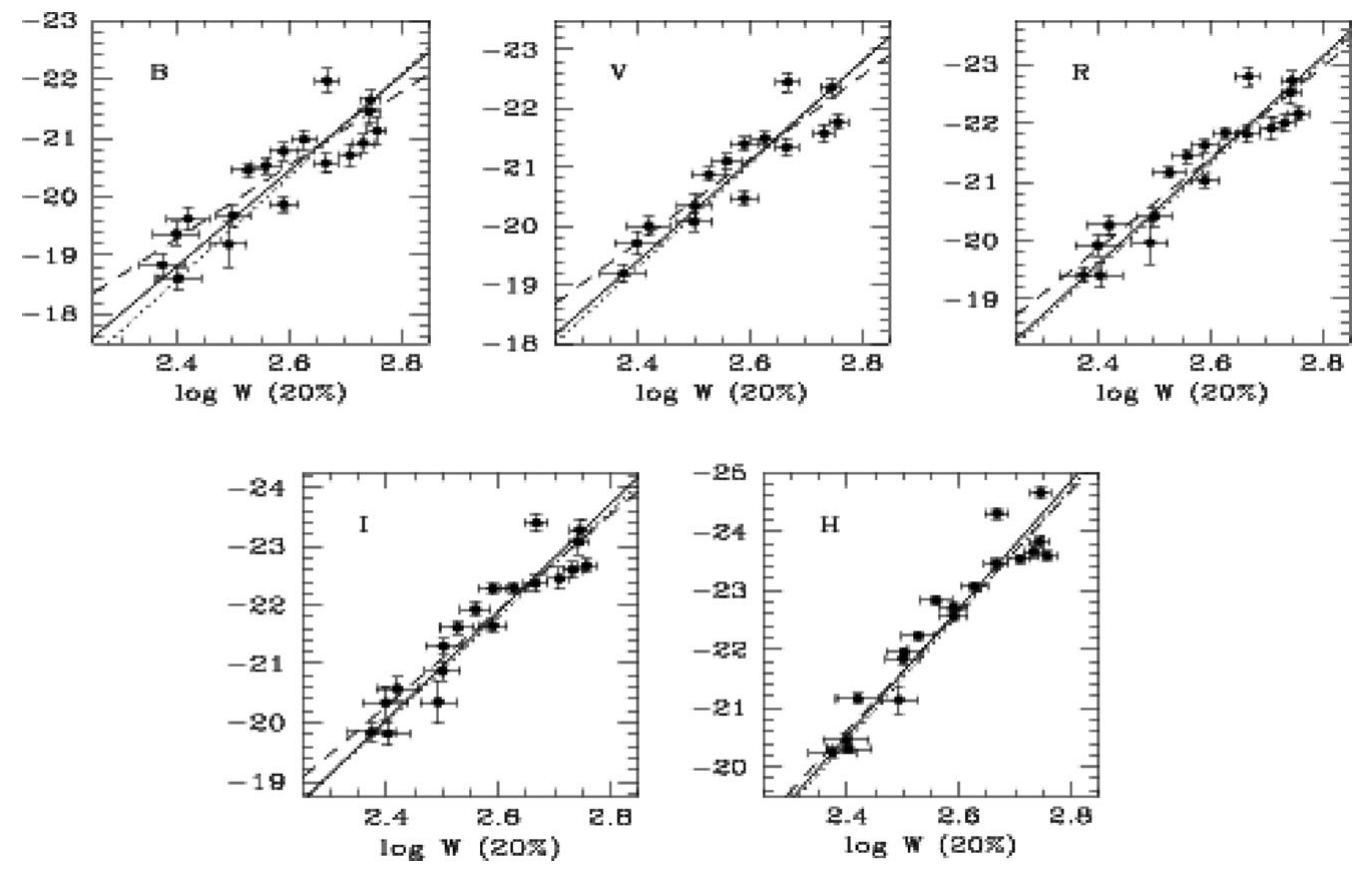

Figure 18. Distances to 21 Cepheid galaxies calibrate the Tully-Fisher relation in the BVRIH bandpasses.

on work from a large number of individuals, including Brent Tully, Riccardo Giovanelli, Mario Hamuy, Mark Phillips, Bob Schommer, Martha Haynes, John Tonry, Adam Riess, Bob Kirshner, Brian Schmidt, Gustav Tammann, Allan Sandage, Mike Pierce, John Blakeslee, George Jacoby, Robin Ciardullo, Sandra Faber, Donald Lynden-Bell, Gary Wegner, David Burstein, Alan Dressler, Roberto Terlevich, Roger Davies and Gerard de Vaucouleurs.

\section{Subsequent Observations and Future Prospects}

A number of important observations have been made since the publication of the Key Project results. The Supernova and $\mathrm{H}_{0}$ for the Equation of State (SHOES) project has carried out a differential analysis of the supernova hosts NGC 4536, 4639, 3982, 3370, 3021 and 1309 with respect to NGC 4258. This eliminates uncertainties such as photometric transformations and crucially, the LMC distance. The maser distance to NGC 4258 is assumed instead. In this way, Riess et al. (2009) obtain $\mathrm{H}_{0}=74.2 \pm 3.6 \mathrm{~km} \mathrm{~s}^{-1} \mathrm{Mpc}^{-1}$. They also find a value of $\mathrm{w}$ in the equation of state $\mathrm{w}=\mathrm{P} / \rho=1.12 \pm 0.12$.

Most importantly, the Hubble Constant has been deduced from the position of the first acoustic peak in the small scale anisotropy of the cosmic microwave background. Given the sound horizon on the surface of last scattering $(143 \pm 4 \mathrm{Mpc})$ and the angular size of the first acoustic peak $(0.601 \pm 0.005)$, one obtains an angular diameter distance for the surface of last scattering of $13.7 \pm 0.4 \mathrm{Gpc}$. Assuming $\Omega_{M}=0.3$ and $\Omega_{\Lambda}=0.7$, this yields a value of $\mathrm{H}_{0}$ of $70 \mathrm{~km} \mathrm{~s}^{-1} \mathrm{Mpc}^{-1}$. Solving for all the cosmological model parameters, Komatsu et al. (2009) find $\mathrm{H}_{0}=70.5 \pm 1.3 \mathrm{~km} \mathrm{~s}^{-1} \mathrm{Mpc}^{-1}$.

Progress will continue in the classical distance scale of the Key Project too. The NASA mission SIM-Lite is expected to yield a rotational parallax from velocities and proper motions for the galaxies M31 and M33 to 1\% accuracy. This will provide a definitive Cepheid period-luminosity relation. 

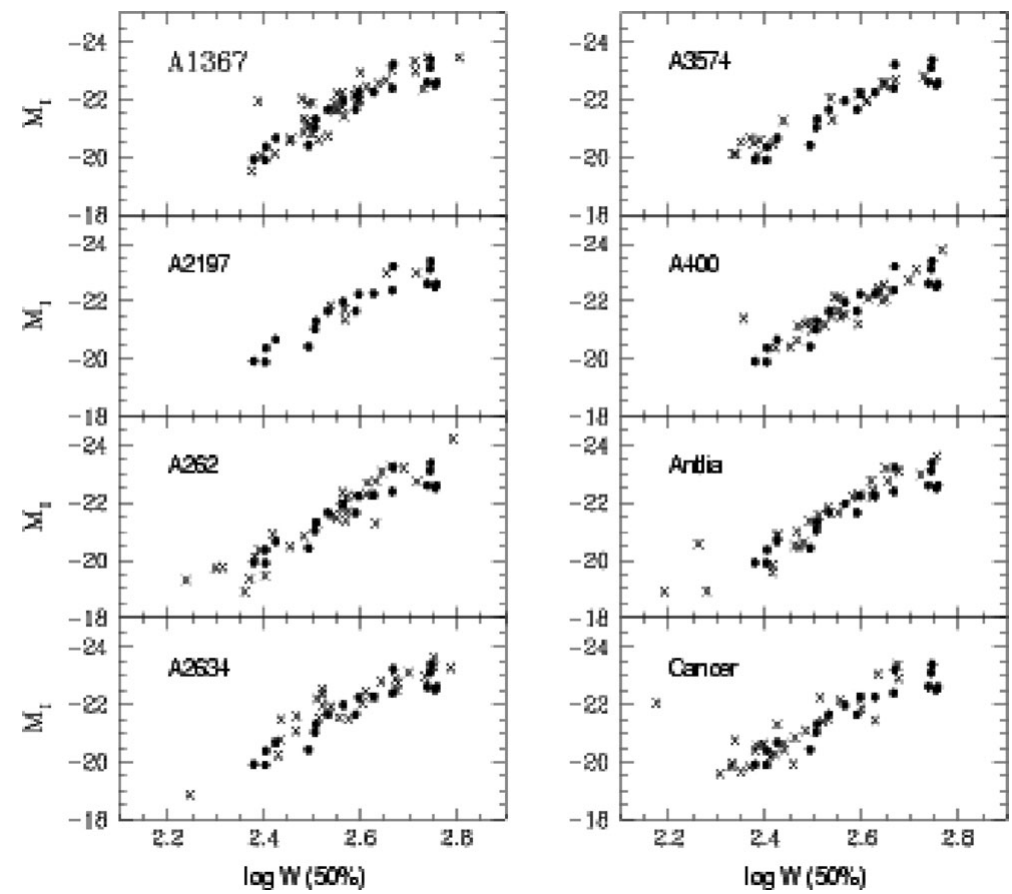

Figure 19. Tully-Fisher distances to clusters of galaxies yield the Hubble Constant.
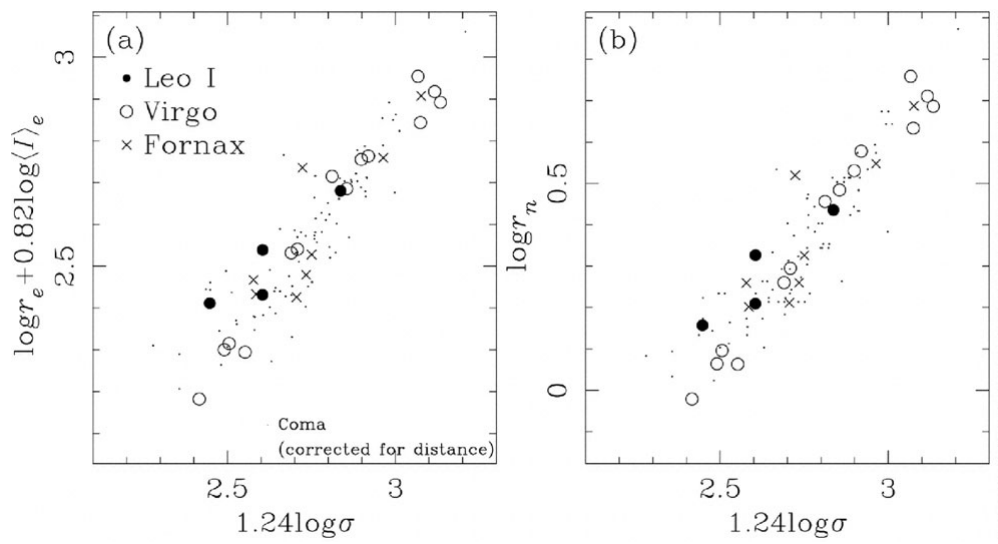

Figure 20. Calibration of the fundamental plane (left) and the $\mathrm{D}_{n}, \sigma$ relation (right). This is superposed on the Coma cluster (dots).

\section{Acknowledgements}

We wish to thank NASA for designating this a Key Project for the Hubble Space Telescope. We wish to thank the team for making it a success. Finally, we wish to thank the Gruber Foundation for recognizing this work.

\section{References}

Benedict, G. et al. 2007, AJ, 133, 1810

Ciardullo, R. \& Jacoby, G. 1992, ApJ, 388, 268

Chiosi, C., Wood, P., \& Capitanio, N. 1993, ApJ (Suppl), 86, 541

Ferrarese, L. et al. 2000a, ApJ, 529, 745 


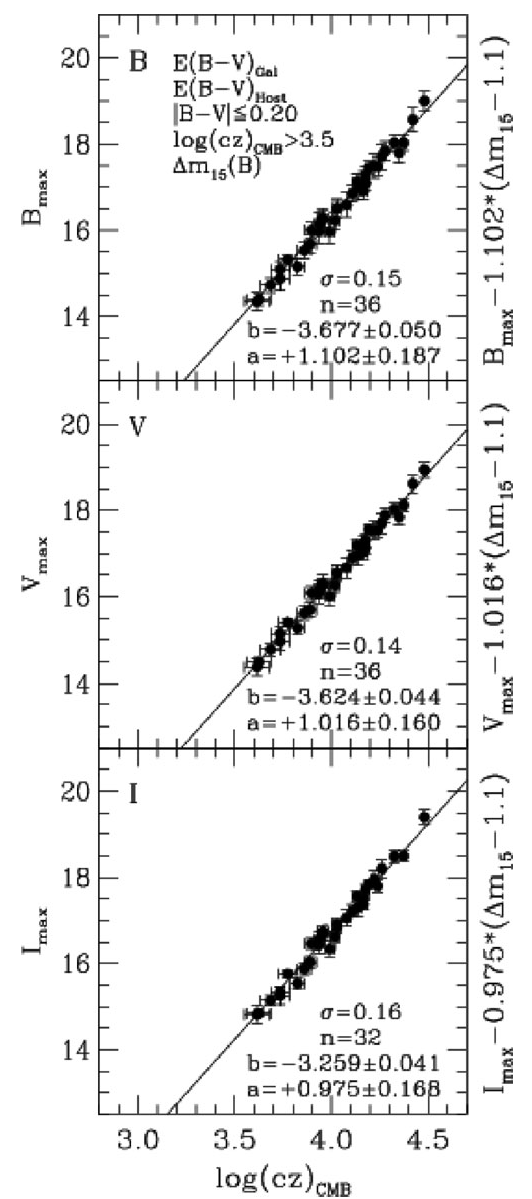

Figure 21. Decline rate corrected supernovae in B,V,I bandpasses showing a dispersion of 0.16 mag.

Ferrarese, L. et al. 2000b, ApJ (Suppl), 128, 431

Freedman, W. et al. 2001, ApJ, 553, 47

Freedman, W. et al. 1991, ApJ, 372, 455

Gibson, B. et al. 2000, ApJ, 529, 723

Herrnstein, R. et al. 1999, Nature, 400, 539

Hubble, E. 1929, Proc. N.A.S., 15, 168

Kelson, D. et al. 2000, ApJ 529, 768

Kennicutt, R. et al. 1998, ApJ, 498, 181

Komatsu, E. et al. 1998, ApJ (Suppl), 180, 330

Leavitt, H. 1908, Ann. Harvard CO, 60, 87

Leavitt, H. \& Pickering, E. 1912, Harvard CO Circ., 173, 1

Macri, L. et al. 2006, ApJ, 652, 1133

Madore, B. \& Freedman, W. 1991, PASP, 103, 933

Madore, B. \& Freedman, W. 2002, SPIE, 4847, 156

Madore, B. \& Freedman, W. 2005, ApJ, 630, 1054

Mould, J. \& Sakai, S. 2008, ApJ, 686, 75

Mould, J. \& Sakai, S. 2009a, ApJ, 694, 1331

Mould, J. \& Sakai, S. 2009b, ApJ, 697, 996

Phelps, R. et al. 1998, ApJ, 500, 763 


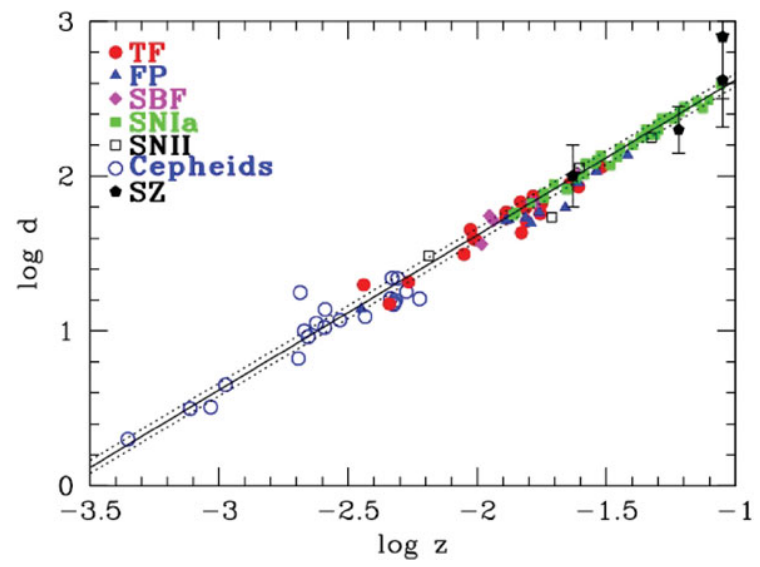

Figure 22. Redshifts and distances for Cepheids, Tully-Fisher clusters, surface brightness fluctuations, the fundamental plane, supernovae of type Ia and type II and clusters with Sunyaev-Zeldovich distances.

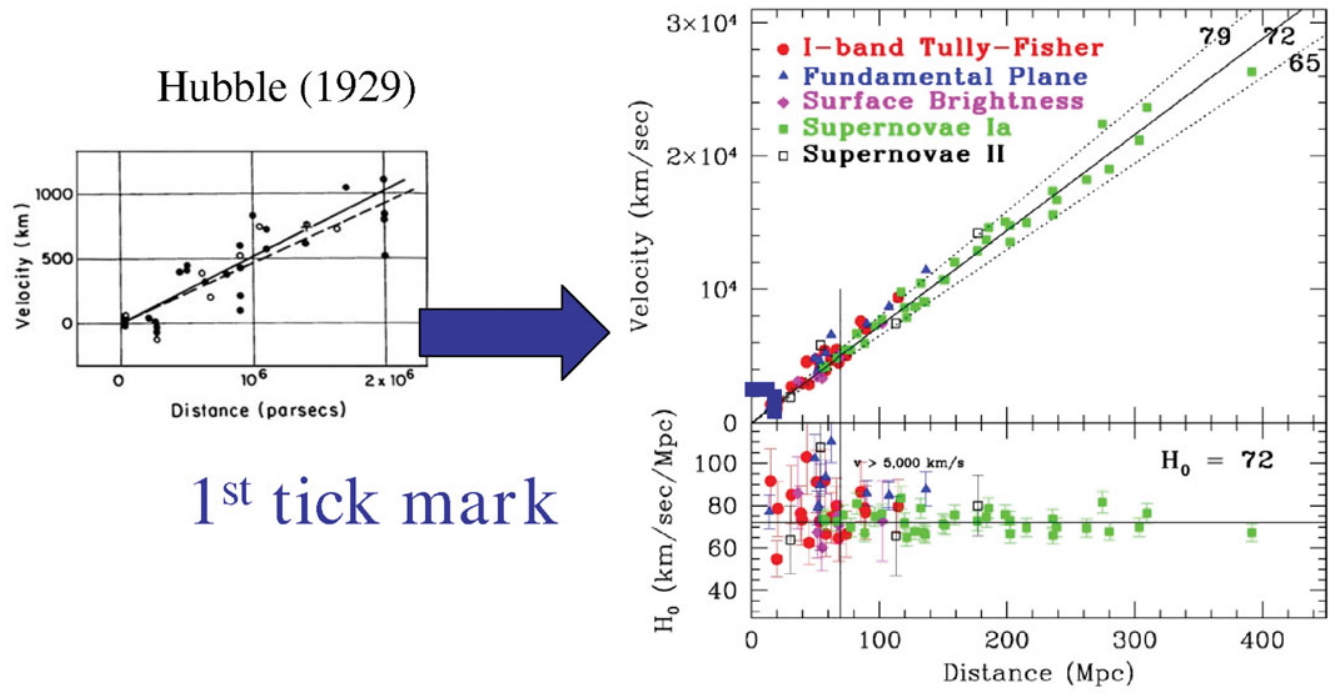

Figure 23. The combined distance indicators yield $\mathrm{H}_{0}=72 \mathrm{~km} \mathrm{~s}^{-1} \mathrm{Mpc}^{-1}$.

Riess, A. et al. 2009, ApJ, 699, 539

Saha, A. et al. 1999, ApJ, 522, 802

Sakai, S. et al. 1997, ApJ, 478, 49

Sakai, S. et al. 2000, ApJ, 529, 698

Sandage, A. et al. 2004, A\&SA, 424, 43

Scowcroft, V. et al. 2009, MNRAS, 396, 1287

Secker, J. \& Harris, W. 1993, AJ, 105, 1358

Stetson, P. 1994, PASP, 106, 250

Tonry, J. et al. 2001, ApJ, 546, 681

Turner et al. 1998, ApJ, 505, 207

Zaritsky, D., Kennicutt, R., \& Huchra, J. 1994, ApJ, 420, 87 


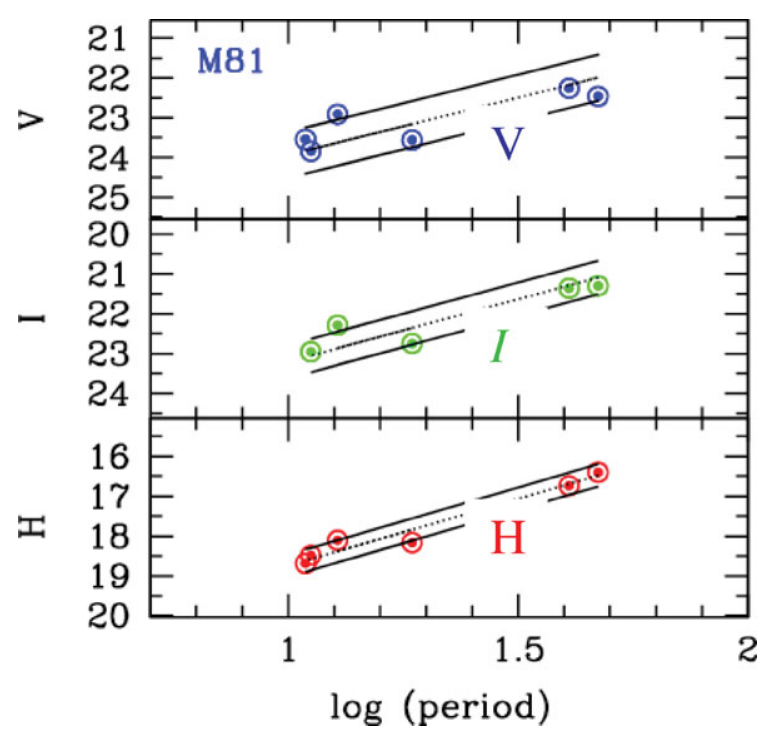

Figure 24. A NICMOS H band period luminosity relation for M81 is compared with Key Project results.

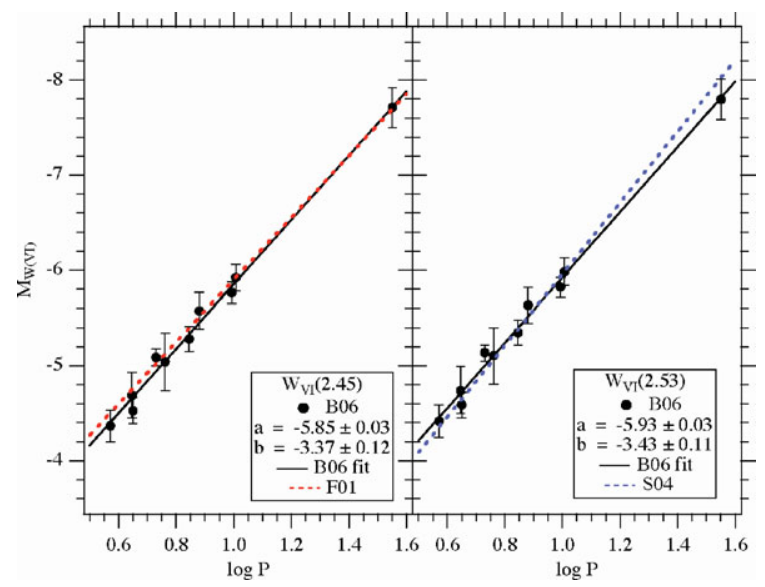

Figure 25. HST parallaxes for Galactic Cepheids yield the period-luminosity relation shown by the filled circles. The Freedman et al. (2001) PL relation is shown on the left and the Sandage et al. (2004) PL relation is shown on the right. The former is a better fit for the periods in excess of 10 days which were used in the Key Project. 


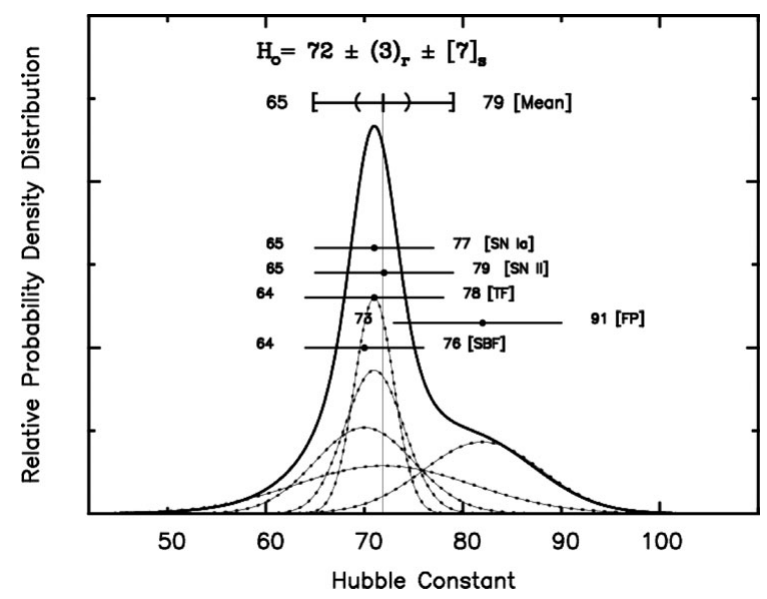

Figure 26. The probability distribution emerging from the Key Project error analysis.

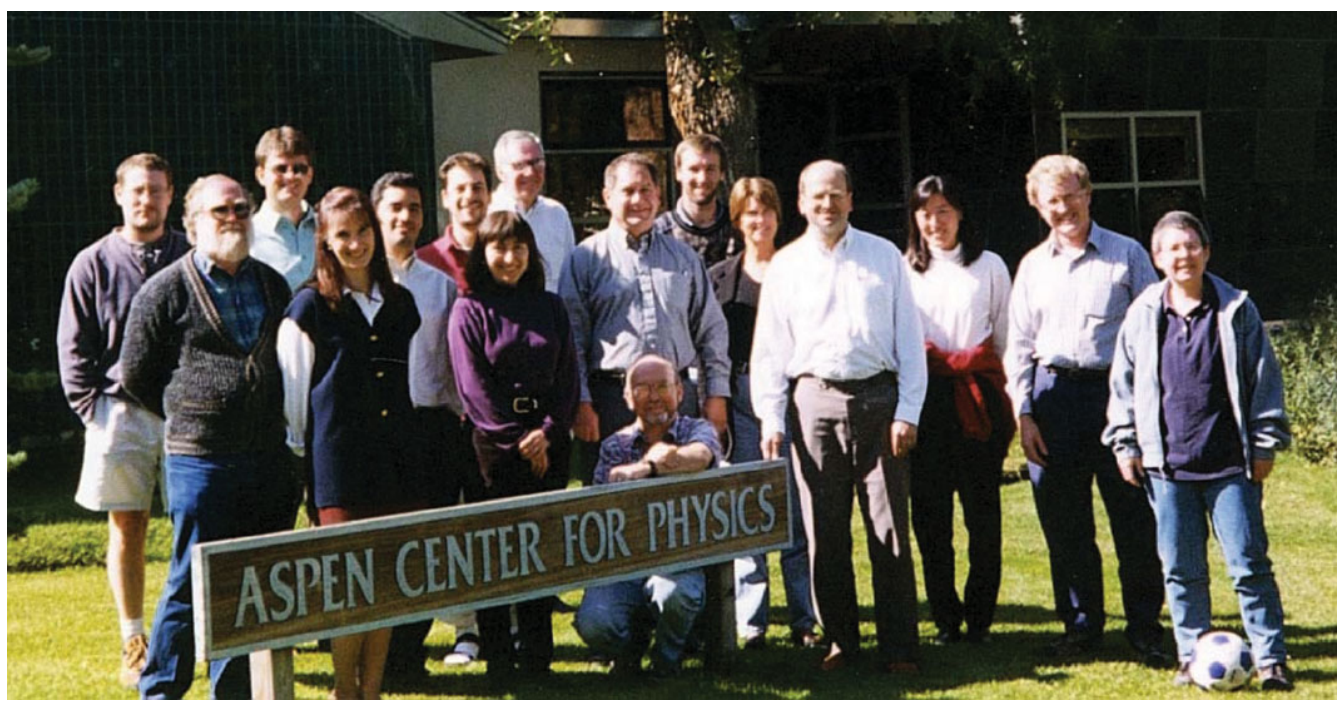

Figure 27. The team photographed at the Aspen Center for Physics in 1999. From left to right: Brad Gibson, Peter Stetson, Shaun Hughes, Laura Ferrarese, Lucas Macri, Dan Kelson, Wendy Freedman, John Graham, Rob Kennicutt, John Huchra, Kim Sebo, Jeremy Mould, Shoko Sakai, Garth Illingworth and Nancy Silbermann 
stellar photometry light curve fitting

Peter Stetson

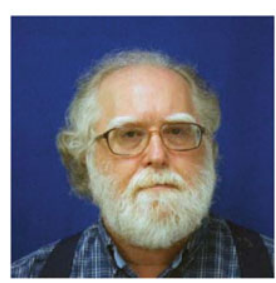

Abi Saha

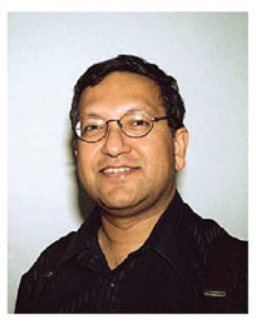

Cepheid observing strategy \& calibration

Figure 28. Special roles were played by three team members.

\section{Shoko Sakai Laura Ferrarese Brad Gibson Dan Kelson}
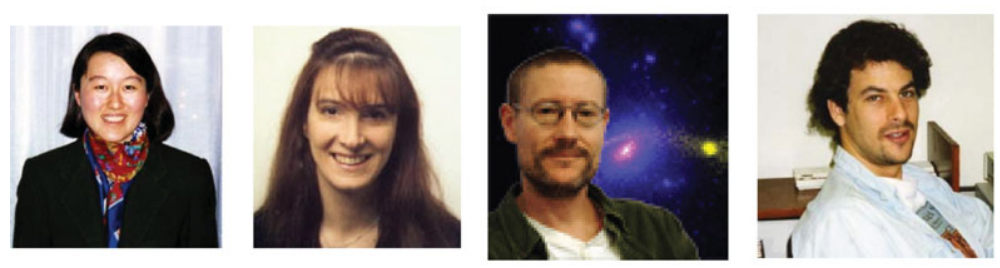

Tully-Fisher relation SBF Type la supernovae $\mathrm{Dn}-\sigma$ relation

Figure 29. Final papers on particular secondary distance indicators were written by four team members. 